\title{
Cuidado em Saúde de adolescentes cumprindo medida socioeducativa
}

\author{
Health Care of adolescents complying with socio-educational measure \\ Atención de salud de adolescentes cumpliendo con medida socioeducativa
}

Recebido: 22/12/2021 | Revisado: 30/12/2021 | Aceito: 01/01/2022 | Publicado: 02/01/2022

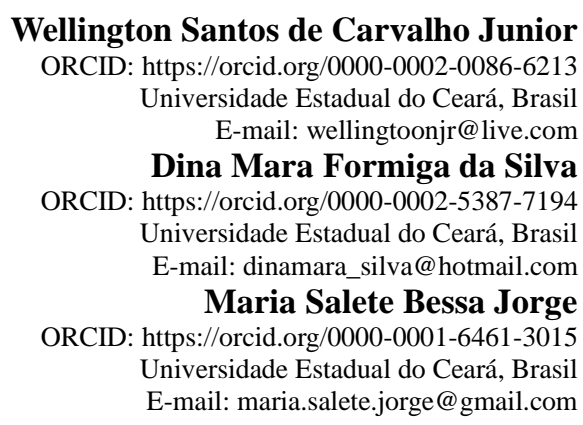

\begin{abstract}
Resumo
A adolescência é um momento importante para a adoção de boas práticas e estabelecimento de autonomia, mas também de exposição a fatores de risco comportamentais, com implicações na saúde em curto e longo prazo. No entanto, os cuidados prestados à saúde no contexto de privação de liberdade ocupam plano secundário, assim como ocorre com as ações de promoção à saúde, sendo ambas essenciais para integrar o adolescente ao contexto social e para promover fatores de proteção e reduzir riscos. Desse modo, esta pesquisa teve como objetivo analisar a percepção dos adolescentes e profissionais inseridos em uma unidade de atendimento socioeducativo sobre os cuidados em saúde na adolescência. Trata-se de um estudo metodológica, de natureza qualitativa, realizado no Centro Socioeducativo São Miguel, participaram da pesquisa, os adolescentes com idade de 15 a 18 anos e profissionais do centro. A análise evidenciou um déficit no conhecimento sobre cuidados em saúde. Considerando, pois, que as unidades de atendimento socioeducativo e as medidas socioeducativas, originalmente concebidas como estratégias de reinserção social, apontam mais para as fragilidades do que para o êxito de seus propósitos, acredita-se que o caráter reparador da aplicação de medida socioeducativa necessita estar associado ao cuidado e à promoção da saúde e da qualidade de vida, a partir da criação de estratégias educativas que possibilitem uma possível modificação de comportamento, gerada por meio de motivação ao adquirir conhecimento sobre determinado tema, associada ao desejo de práticas de vida saudáveis que gerem qualidade de vida.
\end{abstract}

Palavras-chave: Adolescente institucionalizado; Cuidado em saúde; Saúde do adolescente.

\begin{abstract}
Adolescence is an important moment for the adoption of good practices and establishment of autonomy, but also of exposure to behavioral risk factors, with short- and long-term health implications. However, health care in the context of deprivation of liberty occupies a secondary level, as with health promotion actions, both of which are essential to integrate adolescents into the social context and to promote protective factors and reduce risks. Thus, this research aimed to analyze the perception of adolescents and professionals inserted in a socio-educational care unit about health care in adolescence. This is a methodological study of qualitative nature, conducted at the Centro Socioeducational São Miguel, the adolescents aged 15 to 18 years and professionals from the center participated in the research. The analysis showed a lack of knowledge about health care. Considering, therefore, that socio-educational care units and socio-educational measures, originally conceived as strategies of social reintegration, point more to weaknesses than to the success of their purposes, it is believed that the restorative character of the application of socio-educational measures needs to be associated with care and promotion of health and quality of life, from the creation of educational strategies that allow a possible change of behavior, generated through motivation when acquiring knowledge about a given theme, associated with the desire for healthy life practices that generate quality of life.
\end{abstract}

Keywords: Institutionalized adolescent; Health care; Teenager's health.

\section{Resumen}

La adolescencia es un momento importante para la adopción de buenas prácticas y el establecimiento de la autonomía, pero también de la exposición a factores de riesgo conductuales, con implicaciones para la salud a corto y largo plazo. Sin embargo, la atención de la salud en el contexto de la privación de libertad ocupa un nivel secundario, al igual que con las acciones de promoción de la salud, que son esenciales para integrar a los adolescentes en el contexto social y 
promover factores protectores y reducir los riesgos. Así, esta investigación tuvo como objetivo analizar la percepción de adolescentes y profesionales insertos en una unidad de atención socioeducativa sobre la atención de la salud en la adolescencia. Se trata de un estudio metodológico de carácter cualitativo, realizado en el Centro Socioeducativo São Miguel, participaron en la investigación adolescentes de 15 a 18 años y profesionales del centro. El análisis mostró una falta de conocimiento sobre la atención médica. Considerando, por lo tanto, que las unidades de atención socioeducativa y las medidas socioeducativas, concebidas originalmente como estrategias de reinserción social, apuntan más a debilidades que al éxito de sus propósitos, se considera que el carácter restaurador de la aplicación de medidas socioeducativas debe asociarse con la atención y la promoción de la salud y la calidad de vida, desde la creación de estrategias educativas que permitan un posible cambio de comportamiento, generado a través de la motivación al adquirir conocimientos sobre un tema determinado, asociado al deseo de prácticas de vida saludable que generen calidad de vida.

Palabras clave: Adolescente institucionalizado; Atención de la salud; Salud del adolescente.

\section{Introdução}

A adolescência é um momento importante para a adoção de boas práticas e estabelecimento de autonomia, mas também de exposição a fatores de risco comportamentais, com implicações na saúde em curto e longo prazo. Fazendo parte desse processo, a assunção de novos papéis no caminhar para a idade adulta, o qual requer novas experiências, conhecimentos e responsabilidades (Organização Mundial de Saúde, 2003).

Neste cenário, a simples observação do cotidiano e a apreciação da literatura acadêmica revelam que questões político-ideológicas, valores morais e preconceitos comportamentais sobre a adolescência são notáveis. Povoam de forma intensa e arraigada os corações e mentes de gestores e profissionais das áreas da saúde, da educação e do direito, com potencial de consistirem em entraves e, até mesmo, em alguns casos, na inviabilização de iniciativas governamentais voltadas para a melhoria das condições de vida, do bem-estar e da saúde daquele grupo populacional.

A situação se agudiza exponencialmente no que diz respeito ao asseguramento do direito à saúde de adolescentes que cometeram algum ato infracional grave o suficiente para ingressar no sistema socioeducativo e demandar o cumprimento de mdida socioeducativa de internação e/ou internação provisória, conforme a legislação específica para o infrator adolescente.

No Brasil, o Estatuto da Criança e do Adolescente (ECA), instituído pela Lei nº 8.069 de 13 de julho de 1992, define como crianças, as pessoas até doze anos de idade incompletos e como adolescentes aquelas entre mais de doze e dezoito anos de idade incompletos. Crianças e adolescentes estão submetidos a um processo especial de responsabilização quando cometem ato infracional, conduzindo ao cumprimento de medidas socioeducativas em unidades sob a governança dos governos Estaduais (Ministério da Saúde, 2014).

No Ceará, a Superintendência do Sistema Estadual de Atendimento Socioeducativo (SEAS) foi criada pela Lei estadual $n^{\circ} 16.040 / 2016$ e tem como competências a coordenação da gestão e a execução da política de atendimento socioeducativo, conforme o Estatuto da Criança e adolescente (ECA) e as diretrizes do Sistema Nacional de Atendimento Socioeducativo (SINASE) (Ceará, 2016).

A criação desse órgão ocorreu pelas inúmeras denúncias de maus tratos aos adolescentes infratores (PRACIANO, 2018). O reordenamento institucional da SEAS tem como base nos documentos de Direitos Humanos no qual prevê as ações a ser desenvolvidas com adolescentes em conflito com a Lei Estadual nº 16.040/2016 (Ceará, 2016).

Nessa perspectiva foi adotado no atendimento socioeducativo uma ação em que o desenvolvimento do adolescente deve ocorrer de forma integral, contemplando todas as dimensões do ser (biopsicossocial e espiritual) (Ceará, 2016). Garantindo assim acesso a todos os eixos das diretrizes socioeducativas, escolarização, profissionalização, esporte, cultura e lazer. Assim, os centros socioeducativos são capazes de oferecer educação que vai além da educação escolar e profissional, e garantir os direitos à cultura e ao lazer (Ministério da Saúde, 1988).

Conforme nos aponta o Sinase (2006, p. 46) 
O adolescente deve ser alvo de um conjunto de ações socioeducativas que contribua na sua formação, de modo que venha a ser um cidadão autônomo e solidário, capaz de se relacionar melhor consigo mesmo, com os outros e com tudo que integra a sua circunstância e sem reincidir na prática de atos infracionais. Ele deve desenvolver a capacidade de tomar decisões fundamentadas, com critérios para avaliar situações relacionadas ao interesse próprio e ao bem comum aprendendo com a experiência acumulada individual e social, potencializando sua competência pessoal, relacional, cognitiva e produtiva.

Dentro dos eixos do SINASE, que determinam os pilares da socio educação temos o eixo da Educação, profissionalização, esporte, cultura e lazer, segurança, espiritualidade e saúde. Apesar do Estatuto da Criança e adolescente considerar a faixa etária compreendida entre 12 a 18 anos, o Ministério da Saúde considera que a fase se estabelece de 10 a 19 anos conforme a OMS, e para essa faixa etária segundo a saúde é um desenvolvimento prioritário. Segundo o IBGE a adolescência corresponde a $18 \%$ da população segundo censo de 2010. Mas ao mesmo tempo fica em segundo plano apesar de ter um papel relevante na Estratégia de Saúde da Família (ESF) que se destaca dentre umas das Estratégias do SUS.

Dentre as principais barreiras destacam-se a obrigatoriedade de serem acompanhado pelos pais quando vão buscar de atendimento, outro ponto a ser levado em consideração é a falta do conhecimento do serviço onde se vê a necessidade de haver uma necessidade de se encurtar a distância entre o serviço e o público-alvo. Agora imagina a dificuldade do acesso a esses serviços dos adolescentes com conflito com a lei. Muitas das vezes ele precisa estar cumprindo medida socioeducativa em meio fechado para o adolescente ter acesso a algum serviço de saúde, devido à fragilidade e vulnerabilidade social que o adolescente se encontra.

Segundo levantamento do ministério da Mulher, da família e dos Direitos Humanos em 2017 das 484 unidades socioeducativas do país, adolescentes e/ou crianças de 12 a 21 anos de idade. Na data de referência de 30 de novembro 24.803 (vinte e quatro mil e oitocentos e três). Sendo que 17.811 em média de internação (71,8\%), 2.160 em regime de semiliberdade $(8,7)$ e 4.832 em internação provisória $(19,5)$ em atendimento nas 484 unidades voltadas à restrição e privação de liberdade. Onde desse número $96 \%$ representam adolescentes do sexo masculino e apenas $4 \%$ do sexo feminino.

Antes da criação da SEAS no âmbito do estado do Ceará havia muitas questões de violação de direitos dos adolescentes dentro dos Centros socioeducativos do Estado do Ceará.

A crise do socioeducativo no Ceará, motivada, sobretudo, pelo contexto de superlotação e de violação sistemática a direitos humanos dos adolescentes e dos jovens internos às unidades de atendimento socioeducativo do estado, implicou considerável instabilidade neste Sistema e a premência de uma restruturação do atendimento socioeducativo (Carvalho, 2020).

Antes o sistema socioeducativo do Ceará era administrado pela Secretaria do Trabalho e Desenvolvimento Social (STDS), com as várias crises com rebeliões devido a violações de direitos dos adolescentes que no caso houve a interdição de dois centros socioeducativos (Diário do Nordeste, 2014).

Nessa perspectiva com a criação da SEAS, que segue os princípios do SINASE, que consta no Estatuto da Criança e do Adolescente, que foi instituída como uma política pública de Estado, no qual visa articular vário segmentos da sociedade em a fim de se obter um Sistema de Garantia de Direitos. No capítulo V, Seção 1, o SINASE consolida o SUS como órgão garantidor do direito a saúde do adolescente cumprindo medida socioeducativa. A implementação da Política de Atenção Integral à Saúde de Adolescentes e Conflito com a Lei (PNAISARI) vem sendo executado com parcerias com as secretarias municipais a fim de garantir os direitos básico de acesso e atendimento e saúde, com verbas repassadas de forma federal direto para as secretarias, mas que a SEAS, participa como membro e executor das medidas.

Desse modo, esta pesquisa teve como objetivo analisar a percepção dos adolescentes e profissionais inseridos em uma unidade de atendimento socioeducativo sobre os cuidados em saúde na adolescência. 


\section{Metodologia}

O estudo faz parte da pesquisa de mestrado intitulada: Desenvolvimento e validação de vídeo educativo para adolescentes em situação de liberdade privada. É um estudo de natureza metodológica que se constituiu no Centro Socioeducativo São Miguel (CSSS), localizado no bairro Passaré, região sul da cidade de Fortaleza, trata-se de uma unidade de "ressocialização" para adolescente de até dezessete anos, do sexo masculino, em conflito com a Lei, que cumprem medida socioeducativa em regime de internação provisória, enquanto aguardam a conclusão do processo de apuração do ato infracional pelo Juizado da Infância e da Juventude, na Cidade de Fortaleza, Ceará.

A amostra foi censitária, com todos que participam do processo socioeducativo, do Centro Socioeducativo São Miguel, que possui uma representação populacional de 75 adolescentes, equipe técnica, socioeducadores, e adolescentes e da Superintendência Estadual de Atendimento Socioeducativo. Participaram da pesquisa, após assinar o TCLE e TELE, os adolescentes com idade de 15 - 18 anos e profissionais do centro, equipe técnica de nível superior, psicólogos, assistentes sociais e pedagogo e equipe de socioeducadores. Foram excluídos da amostra todos aqueles participantes que não se enquadraram nos critérios de inclusão.

Foram utilizadas as seguintes técnicas de coleta de dados: roda de conversa e entrevista semiestruturada com perguntas relacionadas a conceitos de saúde, higiene pessoal e uso de drogas, a entrevista foi feita com os adolescentes e equipe do CSSM. A Roda de Conversa é uma ferramenta metodológica na qual utilizamos para comunicação dinâmica e produtiva entre diversos grupos. É uma técnica muito rica utilizada na prática de aproximação entre os sujeitos no cotidiano (Afonso; Abade, 2008).

Após a transcrição dos dados colhidos, a análise de conteúdo foi empregada. Na análise o propósito é ir além do descrito, fazendo uma decomposição dos dados e buscando as relações entre partes que foram decompostas e, por último, na interpretação - que pode ser feita após a análise ou após a descrição - busca-se sentido das falas e das ações para se chegar a uma compreensão ou explicação que vão além do descrito e analisado (Minayo, 2014).

O estudo foi aprovado pelo Comitê de Ética em Pesquisa com Seres Humanos da Universidade Estadual do Ceará (UECE), e está de acordo com a Resolução 466/12 do Conselho Nacional de Saúde com o parecer de número: 4.775.685.

\section{Resultados e Discussão}

Os resultados foram agrupados em categorias: Categoria 1 - Conhecimentos dos adolescentes sobre cuidados em saúde. Categoria 2 - Conhecimento dos profissionais sobre a necessidade dos adolescentes tem sobre cuidados em saúde. 
Figura 1 - Resultados agrupados em categorias.

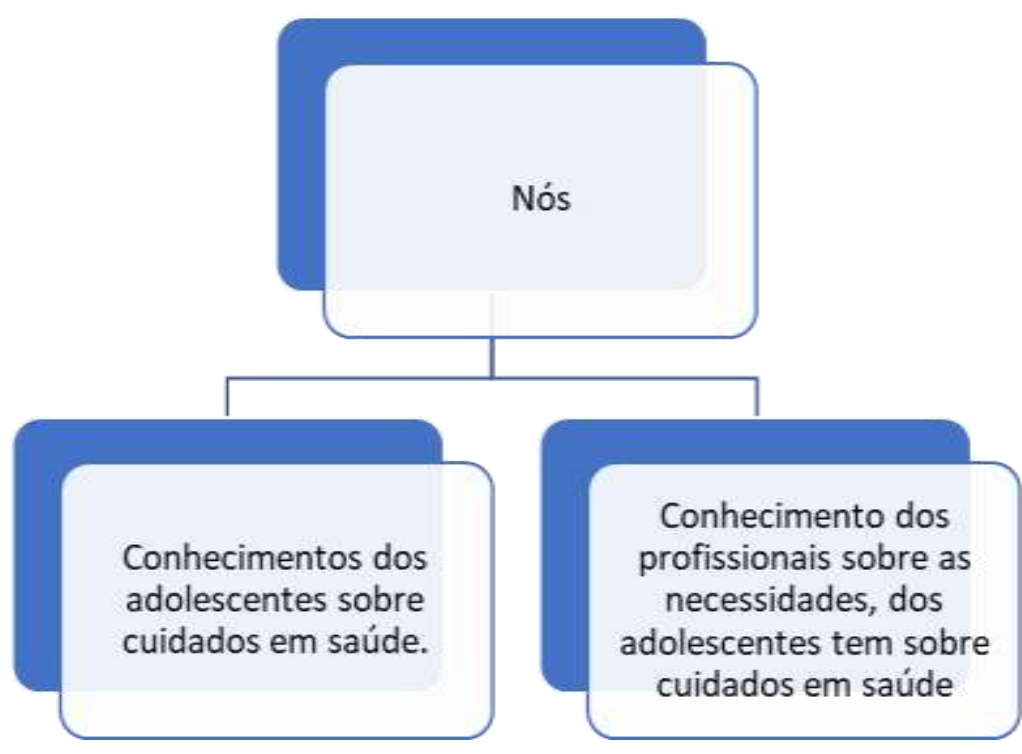

Fonte: Elaborada pelos autores (2021).

Nesta etapa foi realizada análise das falas das entrevistas, as quais foram categorizadas e analisadas para responder às duas perguntas que foram realizadas aos familiares. Os resultados foram efetuados de maneira descritiva.

Categoria 1: Conhecimentos dos adolescentes sobre cuidados em saúde

O termo cuidado em saúde cuidados em relação à prevenção de doenças e promoção da saúde é adotado, para os adolescentes que estão em situação de privação de liberdade, é um assunto de grande relevância. Nós Conhecimentos dos adolescentes sobre cuidados em saúde. Categoria 2 - Conhecimento dos profissionais sobre a necessidade dos adolescentes tem sobre cuidados em saúde onde o adolescente está passando por esse momento que tem sua liberdade tirada, trata-se de um momento complexo e delicado. Tem como proposta tratar problemas sobre a noção de cuidados em saúde que eles têm, a maioria deles vem em um processo de violação de direitos e esse é o primeiro contato que a maioria tem no serviço de saúde.

Figura 2 - Nuvem de palavras.

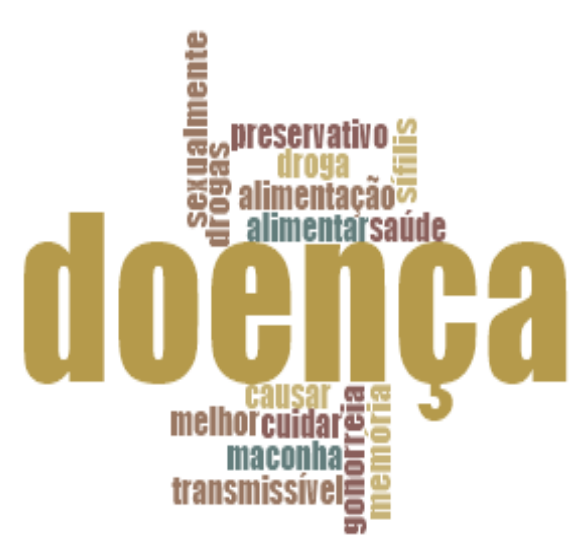

Fonte: Elaborada pelos autores (2021).

Os adolescentes relataram em sua roda de conversa que possuem várias dúvidas sobre o tema proposto. Todos relataram que não tiveram nenhum tipo de orientação em casa, ou em alguma estratégia de saúde da família, o pouco que ainda 
sabem sobre o tema, segundo eles, aprenderam na escola, ou com colegas na rua. Todos se encontravam em situação de abandono escolar, isso fragilizando ainda mais seus conhecimentos sobre o tema.

Todos relatam sobre a importância do uso dos preservativos para evitar as ISTs, falam segundo eles que o uso de drogas "Deixa a pessoa meio baqueada assim memória, tipo amnésia" Os adolescentes relatam também dúvidas sobre Higiene pessoal e sobre a Covid-19, mas seus medos são pelos familiares. Os adolescentes responderam muitas das questões de forma breve, demostrando a falta de conhecimento e como um reflexo de seu baixo processo de escolarização.

Categoria 2: Conhecimento dos profissionais sobre as necessidades, dos adolescentes tem sobre cuidados em saúde

Os profissionais que atuam na linha de frente com os adolescentes que estão cumprindo medida socioeducativa, identificam muito em suas falas as reais necessidades dos adolescentes, pois os mesmos participam diariamente de suas rotinas, atividades e atendimentos, esses relataram que os mesmo quando estão em privação de liberdade, vem com muitos questionamentos e um dos temas mais recorrentes é sobre saúde.

Figura 3 - Nuvem de palavras.

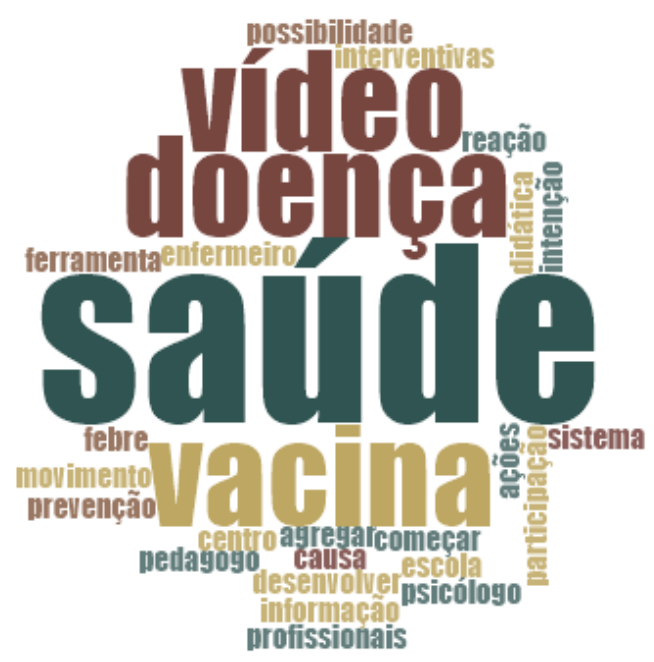

Fonte: Elaborada pelos autores (2021).

A conversa com os profissionais do Centro Socioeducativo São Miguel, se deu muito entorno, de qual seria a melhor estratégia, para a produção de um material, onde os adolescentes poderiam compreender, com uma linguagem clara e objetiva, um pouco destes temas que envolvem o cotidiano deles.

Este estudo revelou que a percepção dos adolescentes acerca do cuidado à sua saúde, perpassa por diversos fatores relacionados ao contexto social dos mesmos e que podem despertar influência nas suas condutas e práticas de saúde. Tais aspectos exercem influência, também, no exercício do autocuidado, bem como na maneira que se vinculam a comunidade escolar, família e profissionais de saúde.

Magalhães (2010), afirma que as entidades e programas do atendimento socioeducativo devem oferecer e garantir aos adolescentes infratores o acesso a programas públicos e comunitários, como por exemplo, escolarização formal, atividades desportistas culturais e de lazer, assistência religiosa, atendimento à saúde na rede pública, inserção em atividades profissionalizantes e inclusão no mercado de trabalho.

Entretanto, os cuidados prestados à saúde no contexto de privação de liberdade ocupam plano secundário, assim como ocorre com as ações de promoção à saúde, sendo ambas essenciais para integrar o adolescente ao contexto social e para promover fatores de proteção e reduzir riscos (Costa, 2007). 
Considerando, pois, que as unidades de atendimento socioeducativo e as medidas socioeducativas, originalmente concebidas como estratégias de reinserção social, apontam mais para as fragilidades do que para o êxito de seus propósitos, acredita-se que o caráter reparador da aplicação de medida socioeducativa necessita estar associado ao cuidado e à promoção da saúde e da qualidade de vida, já que esta é vista como a modalidade mais completa de prevenção ao ato infracional, segundo o ECA.

Corrobora-se com Monteiro (2006) ao afirmar que se pretendemos tornar o adolescente um sujeito responsável consigo e com a sociedade, precisamos oferecer ferramentas que potencializem o aprendizado e o seu desenvolvimento integral. Assim, a atenção integral à saúde do adolescente em cumprimento de medidas socioeducativas, de restrição e privação de liberdade, implica numa atenção com a máxima integralidade e com um atendimento singular as suas demandas especificas, tanto no que concerne ao período específico da vida, como no contexto ao qual o adolescente está inserido.

Nessa perspectiva, a tecnologia educacional surge como ferramenta para modificar o paradigma de ação educativa no cuidado e promoção à saúde, pois contempla critérios de uma aprendizagem efetiva, e o conhecimento gerado a partir da tecnologia pode ser transportado para o campo da realidade.

Ante este contexto, torna-se pertinente, criar estratégias educativas que possibilitem uma possível modificação de comportamento, gerada por meio de motivação ao adquirir conhecimento sobre determinado tema, associada ao desejo de práticas de vida saudáveis que gerem qualidade de vida.

A velocidade das transformações na sociedade contemporânea marcada pela produção desenfreada de conhecimentos científicos, pelas mudanças dos valores, pela influência dos meios de comunicação na formação do homem e do profissional tem implicação direta sobre o pensar-fazer em saúde, demandando assim, a necessidade de refletir sobre o processo de ensinoaprendizagem (Mitre et al., 2008).

Desse modo, ponderar sobre a justificativa dessa propositura, inclui a produção sobre tecnologias leves para a educação em saúde, com o intuito de agir eficazmente na prevenção e promoção à saúde de adolescentes em regime de internação e semiliberdade. Diante deste fato, faz-se necessário utilizar de tecnologias educacionais pois estas são mais acessíveis e eficientes após validação e testes (Oliveira; Fernandes; Sawada, 2008).

Foi feito um benchmarking para aprofundar-se de todo conteúdo relativo a educação em saúde na socioeducação através de uma busca detalhada além de revistas e periódicos científicos será buscado dentro das secretarias e órgãos que gerem o sistema socioeducativo no Brasil. Para organização da seleção e busca de conteúdos educacionais será elaborado checklist com a base na metodologia Prisma. O processo de seleção dos estudos normalmente é conduzido por meio do ckecklist Prisma - Preferred Reporting Items for systematic reviews and Meta-Analyses (MOHER et. al., 2009).

Nesse contexto, os recursos audiovisuais, a exemplo de vídeos disponíveis na internet, se apresentam como fortes aliados para facilitar o ensino/aprendizagem sobre cuidados em saúde. O uso do vídeo educativo apresenta-se como um instrumento didático e tecnológico, constituindo-se em uma ferramenta que proporciona conhecimento, favorece a consciência crítica e a promoção da saúde (Razera et al., 2014).

Nessa ótica, Pimenta et al. (2007) destacam que as imagens exibidas nos vídeos podem ir muito além da simples transmissão da informação; pensar novos rumos pedagógicos e experimentais pode levar a uma ampliação do horizonte teórico e metodológico de cada um, gerando uma verdadeira práxis do audiovisual e da imagem no campo da saúde.

Consoante Gómez e Pérez (2013) relatam que os vídeos educativos têm sido utilizados em diversas experiências pedagógicas demonstrando a relevância da sua aplicabilidade no processo de ensino aprendizagem, pois combinam vários elementos, tais como imagens, texto e som em um único objeto de promoção do conhecimento. Esta prática é essencial aos profissionais de saúde, que devem utilizar deste recurso para promover saúde, troca de conhecimentos, além de padronizar ações que contribuirão na ressignificação das condições de saúde tornando um importante aliado na prática profissional. 
Justifica-se a pesquisa pela existência de uma lacuna na produção de conhecimento sobre a integralidade das ações de saúde para os adolescentes que cumprem medidas socioeducativas, ao mesmo tempo em que, estudos nessa linha, representam oportunidades de conhecer de maneira mais consistente e apropriada os frutos e resultados da importância da promoção da saúde na qualidade de vida das pessoas.

Destarte, emerge a necessidade de medidas educativas por meio de recursos audiovisuais que facilitem e otimizem a compreensão sobre a importância de temas como as doenças sexualmente transmissíveis, álcool e drogas, violência, hábitos alimentares saudáveis, prática de atividade física. Na medida em que essa prática pode favorecer o desencadeamento de processos internos de revisão de valores e posturas, podem ser consideradas como instrumento valioso na prevenção e promoção da saúde.

\section{Considerações Finais}

As entrevistas de grupo permitiram ouvir os adolescentes e os profissionais de saúde que inseridos em um Centro Socioeducativo, dando-lhes a oportunidade de informar suas percepções sobre a importância dos cuidados em saúde na adolescência. Conhecer o que pensam, sentem e preferem os adolescentes sobre os cuidados de saúde, permite compreender melhor as suas perspectivas e saber como adequar os cuidados aos seus desejos, necessidades e preferências, no sentido de estimular o seu interesse e motivação pela procura deles, e não apenas em condição de doença.

Portanto, o conjunto dos dados sugere a necessidade de mudanças nas práticas organizacionais e, particularmente, no desenvolvimento das políticas públicas de saúde, e vem reforçar a necessidade da implementação do que está preconizado nas políticas de saúde para adolescentes, no sentido de tornarem-se mais ajustadas às suas necessidades específicas e preferências e, permitindo uma contribuição e um maior envolvimento dos adolescentes na sua própria saúde.

\section{Referências}

Afonso, M. L. M. \& Abade, F. L (2008). Para reinventar as rodas. Recimam.

Alexandre, N. M. C. \& Coluci, M. Z. O. (2011). Validade de conteúdo nos processos de construção e adaptação de instrumentos de medidas. Ciênc. saúde coletiva, Rio de Janeiro. 16(7), 3061-8.

Areas Neto, N. T., Constantino, P., \& Assis, S. G. (2017). Análise bibliográfica da produção em saúde sobre adolescentes cumprindo medidas socioeducativas de privação de liberdade. Physis, 27(3), 511-540.

Asquidamini, F., Barbiani, R., \& Sugizaki, E. (2015). Adolescentes em medida socioeducativa: violência ou violação de direitos? Revisão de literatura. Rev. Subj., 15(1), 113-123.

Bardin, L. (2010). Análise de conteúdo. Edições 70.

Brasil. (2012). Presidência da República. Secretaria Especial dos Direitos humanos. Conselho Nacional dos Direitos da criança e do Adolescente. Sistema Nacional de Atendimento Socioeducativo - SINASE. Brasília: CONADA".

Brasil. (2014). Secretaria Geral da Presidência da República. Mapa do Encarceramento. Brasília: Secretaria Geral da Presidência da República.

Ceará (Estado). (2016) Lei Estadual no 16.040 de 28 de junho de 2016. Cria a Superintendência do Sistema Estadual de Atendimento Socioeducativo, os cargos de Superintendente e Superintendente Adjunto, o Conselho Gestor da Superintendência, cargos efetivos, a comissão para a elaboração do Plano Estadual Decenal de Atendimento Socioeducativo, institui e autoriza a concessão de gratificações. Diário Oficial do Estado do Ceará, Fortaleza.

Costa, C. R. B. S. F. (2007). Contexto socioeducativo e a promoção de proteção a adolescentes em cumprimento de medida judicial de internação no Amazonas. Rio de Janeiro, 2007. 234 f. Tese (Doutorado em Saúde Pública) - Escola Nacional de Saúde Pública, Fiocruz.

Diário do Nordeste. (2017). Vigilante de escola é morto em serviço. Fortaleza: Diário do Nordeste.https://diariodonordeste.verdesmares.com.br/seguranca/vigilante-de-escola-morto-em-servico-1.1737095.

Filatro, A., \& Cairo, S. (2015). Produção de conteúdos educacionais. . Saraiva.

Kinner, S. A. et al. (2020). The health of children deprived of liberty: a human rights issue. The Lancet Child \& Adolescent Health, 4(1), 6-7.

Lage, M. C. (2010) Utilização do software Nvivo em pesquisa qualitativa: uma experiência em EaD. ETD - Educação Temática Digital, $12(1)$, $198-226$. 
Research, Society and Development, v. 11, n. 1, e9711125086, 2022

(CC BY 4.0) | ISSN 2525-3409 | DOI: http://dx.doi.org/10.33448/rsd-v11i1.25086

Leite, S. de S., et al. (2018). Construction and validation of an Educational Content Validation Instrument in Health. Revista Brasileira de Enfermagem, 4 , $1635-1641$.

Minayo, M. C. S. (2007). Pesquisa social: teoria, método e criatividade. (21a ed.), Vozes, 20.

Ministério da Saúde. (1990). Lei no 8.069, de 13 de julho de 1990. Dispõe sobre o Estatuto da Criança e do Adolescente, e dá outras providências. Diário Oficial da União, Brasília, DF, 13 jul. 1990. Seção 1, p. 1.

Mitre, S. M. et al. (2008). Metodologias ativas de ensino-aprendizagem na formação profissional em saúde: debates atuais. Ciênc. saúde coletiva. 13(2), 21332144.

Organização Mundial da Saúde. (2003). CID -10, tradução do centro colaborador da OMS para a classificação de doenças em Português. 9. ed. São Paulo: EDUSP.

Perminio, H. B., et al. (2018). Política Nacional de Atenção Integral à Saúde de Adolescentes Privados de Liberdade: uma análise de sua implementação. Ciênc. saúde coletiva, 23(9), 2859-2868. 